\title{
Development and Validation of an Alternate Stability- indicating UV Spectrophotometric Analytical Method for Aspirin in Tablets
}

\author{
M. DOLORES, E. A. MORALES-HIPÓLITO, J. A. GARDUÑO-ROSAS, A. VILLASEÑOR AND R. LÓPEZ-ARELLANO*
}

Laboratory of Pharmaceutical Development Tests, Multidisciplinary Research Unit (UIM), Faculty of Higher Education Cuautitlán, National Autonomous University of Mexico, Carr. Cuautitlán-Teoloyucan Km 2.5, San Sebastian Xhala, 54714 Cuautitlán Izcalli, Edo. of México

Dolores, et al.: Alternate Spectrophotometric Method for Determination of Aspirin

\begin{abstract}
Aspirin is a drug that has been used for widely for more than a century. Numerous stability-indicating analytical methods have been reported over the years. A new stability indicating method was developed for the accurate quantitation of aspirin in tablets using the partial least squares calibration algorithm. This method is specific for the determination of aspirin and its main degradation product; salicylic acid in tablets using ultraviolet spectrophotometry. The calibration matrix needs only 13 binary solutions and the method showed good linearity from 30 to $70 \mu \mathrm{g} / \mathrm{ml}$ for aspirin and 0 to $40 \mu \mathrm{g} / \mathrm{ml}$ for salicylic acid. Moreover, a full validation according to the International Council of Harmonization guidelines was carried out covering precision, recovery, sensitivity, sample stability and tolerance. The method was applied to developed and commercial aspirin tablets. The proposed method represents a reliable choice to be used as a routine method for quality control of newly manufactured products.
\end{abstract}

Key words: Aspirin, stability-indicating method, partial least squares, UV spectrophotometry, validation

Aspirin (ASP) also known as acetylsalicylic acid is a well-established drug used worldwide as an and analgesic, antipyretic and antiinflammatory drug ${ }^{[1-3]}$. ASP along with its main and active degradation product salicylic acid (SA) has been studied for decades. Basically, once ASP is ingested, it is immediately hydrolysed to $\mathrm{SA}$, which is responsible for the pharmacological activity (fig. 1) ${ }^{[4]}$. This apparently simple reaction has been studied in depth over the years and currently all metabolites from ASP intake along with their interaction inside the body are well known ${ }^{[5]}$.

In addition, a literature survey exposed there were numerous analytical methodologies to detect ASP and SA in tablets and biofluids ${ }^{[6-17]}$. Particularly, for the pharmaceutical industry, the interest raises on the need of developing robust analytical stability methods to ensure drugs will not degrade until the sell-by date has expired ${ }^{[18,19]}$. Ideally, a stability indicating method (SIM) should be able to distinguish between any degradation product from active substance keeping an accurate and precise quantitation of both ${ }^{[20]}$.

For ASP stability monitoring in tablets, ultraviolet

${ }^{*}$ Address for correspondence

E-mail: lopezar@unam.mx

November-December 2016

Indian Journal of Pharmaceutical Sciences
(UV) detector has been found to be fit for the purpose as it is suitable to accurately measure in the microgram order, therefore most of the methodologies reported previously employed high performance liquid chromatography (HPLC) technique ${ }^{[7,13,21-23]}$. However, with the introduction of multicomponent methods, UV spectrophotometry showed an astonishing capability in the quantitation of compounds in mixtures without previous separation ${ }^{[8-11,15-17,24]}$. This aspect revealed great advantage over HPLC methods, such as lower cost, less time of analysis and minimum organic solvent waste. This method has been applied for ASP alone or in combination with other drugs, however to our knowledge there is no method employed partial least square (PLS) algorithm. This is peculiarly important because PLS method has become the most common chemometric tool in multivariate analysis.

This is an open access article distributed under the terms of the Creative Commons Attribution-NonCommercial-ShareAlike 3.0 License, which allows others to remix, tweak, and build upon the work non-commercially, as long as the author is credited and the new creations are licensed under the identical terms

Accepted 29 November 2016

Revised 20 October 2016

Received 31 May 2016

Indian J Pharm Sci 2016;78(6):810-817 


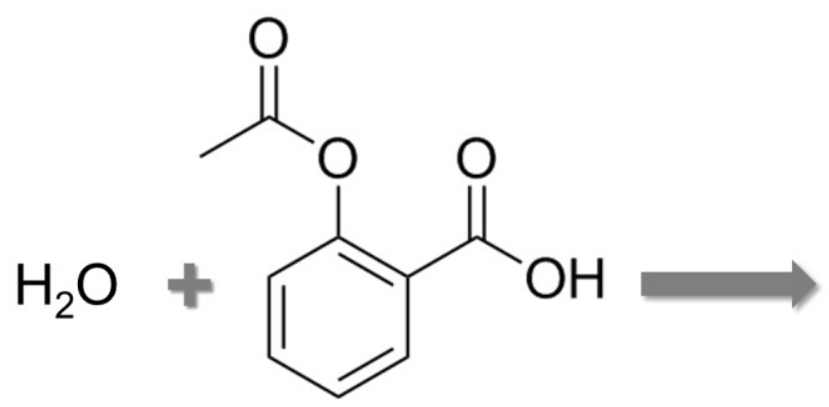

ASP

Fig. 1: Principal degradation reaction of ASP.

Aspirin (ASP); salysilic acid (SA)

This is a very robust and reliable tool that allows the simultaneous quantitation of various components in a sample which commonly contains chemical interferences ${ }^{[24]}$. The PLS model works through a calibration matrix where analytes of interest are mixed in different proportions. The PLS regression model transforms analyte standard concentrations and their resultant absorbance values from a data matrix into latent variables based on a mathematic algorithm, which later predicts the concentration of the analytes from a new sample with high accuracy. Based on the advantages described, the present study shows the development and a full validation of an analytical SIM method using PLS multivariate regression to quantify the content of marketed and developed ASP tablets. This work enhances the repertoire of analytical methodologies employed in the quantitation of ASP in tablets and highlights the advantage of avoiding all kind of separation technique to obtain accurate determinations.

\section{MATERIALS AND METHODS}

AAP (100\% pure) was purchased from Helm Pureza (Hamburg, Germany) and SA 99.5\% was from SigmaAldrich (Milan, Italy). Aspirin ${ }^{\circledR}$ commercial tables containing $500 \mathrm{mg}$ of ASP were obtained from Bayer Pharmaceutical Company (Mexico City, Mexico, Batch no. X207UU). Raw material and excipients were obtained from Laboratories "Helm de México" (Mexico) and anhydrous alcohol reagent grade was from J.T. Baker. Anhydrous alcohol was the solvent used for all the solutions preparation, since it is well known for the hydrolysis of ASP on aqueous media.

A double-beam system Cary Win UV/Vis, model Cary 1 E Scanning Spectrophotometer (Varian, California, USA), equipped with $1 \mathrm{~cm}$ quartz cell was used.

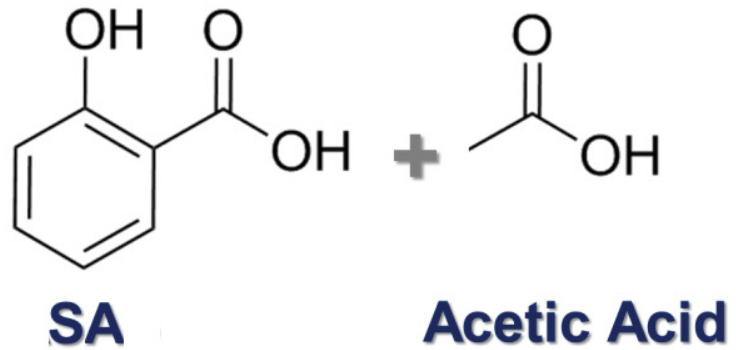

Absorption spectra and simple runs were recorded over the wavelength range of 200-350 nm against the blank using anhydrous alcohol. All the measurements were obtained at room temperature and ambient humidity. Data was recollected using the programs 'Cary simple reads' and 'Cary scan' in ASCII format.

\section{Calibration mix:}

Standard solutions for the calibration mix were prepared from independent stock solutions of ASP and SA (25 $\mathrm{mg} / 100 \mathrm{ml}$ ) in anhydrous alcohol. Standard series of solutions containing ASP from 30 to $70 \mu \mathrm{g} / \mathrm{ml}$ and SA from 0 to $40 \mu \mathrm{g} / \mathrm{ml}$ were obtained accomplishing a combination set of 13 mixture systems by triplicate (fig. 2). The calibration solutions were prepared freshly every day from stocks.

\section{PLS multivariate regression method:}

The mathematical PLS multivariate algorithm is detailed elsewhere ${ }^{[25]}$. Basically, in order to apply the PLS regression, a calibration matrix containing standard binary solutions has to be performed. These solutions have to be measured at different wavelengths, and their absorption has to be noted on a data matrix. These data is pretreated by resting the average of the concentration and absorbance at each wavelength to each original data, this step is called centring and normalization. Using this transformed matrix, the PLS algorithm is applied; hereby the resultant regression algorithm creates a PLS model that might estimate the concentration of analytes in unknown samples. The quality of the resultant model is assessed by the prediction capability $\left(\mathrm{R}^{2}\right)$. The complete description of this methodology is presented in fig 2. Multivariate data treatment was carried out by Datan 5.0.4.4 statistical program (Copyright Multid Analyzes AB). 


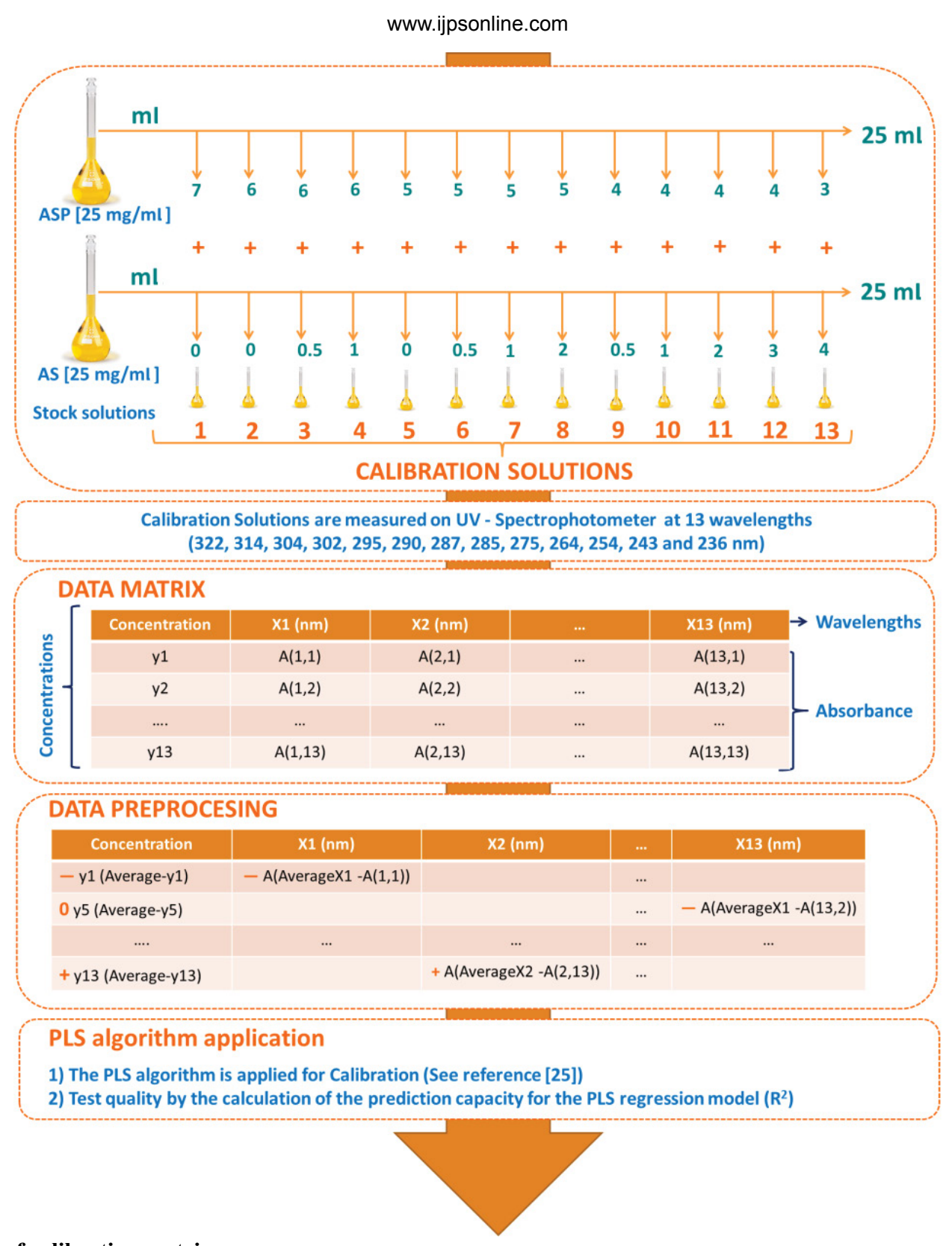

Fig. 2: Workflow of calibration matrix.

The model obtained from the last step is used to calculate unknown concentrations of ASP and SA on new samples.

\section{Sample preparation:}

Twenty tablets were weighed and powdered in a mortar until a uniform powder was obtained. Approximately $60 \mathrm{mg}$ of tablet containing the equivalent amount of 50 $\mathrm{mg}$ of ASP was weighed and transferred to a $100 \mathrm{ml}$ beaker where a volume of $60 \mathrm{ml}$ of anhydrous alcohol was added. The sample was dissolved and stirred with magnetic agitation for $5 \mathrm{~min}$ at $60 \mathrm{rpm}$. After complete dissolution, the sample was filtered using Whatman 40 filter paper. The filtrate was collected and filled up to the mark in a $100 \mathrm{ml}$ volumetric flask using anhydrous alcohol (solution A). Second dilution was performed using an aliquot of $5 \mathrm{ml}$ from solution A, and diluted with anhydrous alcohol to the mark in a $50 \mathrm{ml}$ volumetric flask (solution B). Resulting solution was in a concentration of $50 \mu \mathrm{g} / \mathrm{ml}$.

\section{Validation study:}

The UV/Vis spectrophotometry methodology using PLS multivariate regression, was validated accordingly to ICH guidelines ${ }^{[26,27]}$ with emphasis on specificity, linearity, precision, accuracy, sensitivity, tolerance and robustness. In particular, specificity was demonstrated by comparing the slope of a calibration curve using standards and spiked samples and through the significant analysis using analysis of 
variance (ANOVA) of concentrations sets obtained by interpolation of values from one calibration curve on the complementary curve and vice versa.

Linearity was estimated by assaying at least five levels of concentrations in mixture of ASP and SA in triplicate, covering values ranges from 60 to $140 \%$ for ASP $(30-70 \mu \mathrm{g} / \mathrm{ml})$ and from 0 to $80 \%$ for AS $(0-40 \mu \mathrm{g} /$ $\mathrm{ml})$. For samples, spiking was performed in 5 different proportions of ASP:SA (110:0, 100:0, 90:10, 80:20, 70:30\%), respectively. Precision and recovery were checked by injecting mixture solutions of ASP and SA standards and spiked samples (120:10, 100:0, 80:40\% of ASP:SA) by sextuple. Within-day and inter-day precision was evaluated on the spiked samples prepared at the $100 \%$ ASP of linearity by two analysts on different days. Furthermore, sensitivity was estimated using 7 levels of ASP and SA very close to each other in concentration by triplicate using standards whereas 9 levels were proposed for spiked samples. Sensitivity was calculated using least significant difference (LSD) statistical test.

Tolerance was estimated measuring three replicates of ASP $100 \%$ in spiked samples at the established wavelengths and 0.2 units above. The robustness was also determined by estimating the influence by the change of medium dissolution to methanol. In this case, absorption spectra of ASP and SA were obtained to observe any change in the systems.

\section{ASP degradation after sample preparation:}

Degradation of prepared samples was checked over the time using standard binary solutions, developed and Aspirin ${ }^{\circledR}$ marketed tablets. For standards, six replicates of ASP:SA standards mixes (120:10, 100:0, 80:40\% of ASP:SA ratio) were analysed every $3 \mathrm{~h}$ until $22 \mathrm{~h}$ after sample preparation.

\section{RESULTS AND DISCUSSION}

Information about ASP and SA was obtained from absorption spectrum of each compound alone and mixed, all dissolved in absolute alcohol (fig. 3). The results showed there was a strong overlapping in almost all wavelengths of the spectra. Therefore on a traditional way of analysis, a direct determination of ASP and SA in a pharmaceutical sample could not possible without a previous separation of analytes. Consequently multivariate regression was tested as an excellent choice to achieve the quantitation of both drugs without the necessity of separation equipment.
The wavelength selection for the PLS regression was done choosing those wavelengths with greater sensitivity to concentration and those where analyte showed a characteristic absorption behaviour in the spectrum compared to other compounds of the mix including excipients. Following these criteria (fig. 3), wavelength range from 200 to $236 \mathrm{~nm}$ was avoided, because over this range it is very likely to observe an interaction with any excipient. From 236 to $295 \mathrm{~nm}$ range, ASP showed higher absorption than SA, and had a maximum signal at $275 \mathrm{~nm}$. Therefore, this range was optimum to select some wavelengths for the model. The range from 295 to $350 \mathrm{~nm}$ only SA showed signal with a maximum at $304 \mathrm{~nm}$ hence some wavelengths were also selected at this range. Herein, continuous mathematical simulations were performed; this means that PLS algorithm was applied to different data matrix, at first using all absorbance wavelengths from the spectrum, and then applying the criteria previously commented. The final regression PLS model was set until the most reliable and straightforward model was obtained considering minimum number of wavelengths. This optimization was based on the accuracy of the PLS to predict the real concentration of standard solutions containing ASP and SA, and checking there was no bias in the estimation. After optimization, 13 was the minimum number of wavelengths for the calibration matrix, these were; 322, 314, 304, 302, 295, 290, 287, $285,275,264,254,243$ and $236 \mathrm{~nm}$. Of note, in the first four wavelengths ASP does not showed absorbance, which meant that for single quantitation of ASP, it would be necessary only 9 wavelength measures.

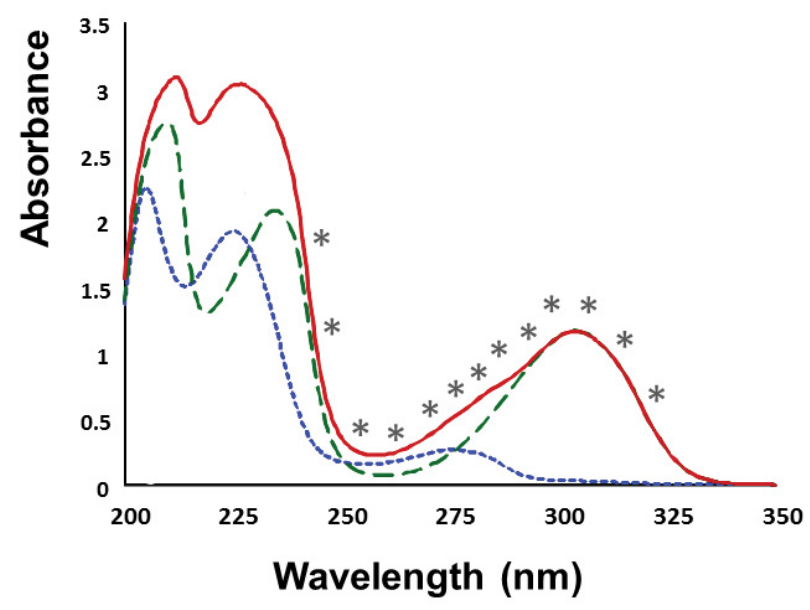

Fig. 3: Comparison of absorption spectra in anhydrous alcohol media.

A) Aspirin $32 \mu \mathrm{g} / \mathrm{ml}$ (ASP), B) salicylic acid $32 \mu \mathrm{g} / \mathrm{ml}$ (SA) and C) standard mixture (ASP+SA). *Selected wavelengths for ASP and SA calibration curves. $---\mathrm{ASP} ;--\mathrm{SA}$; ASP+SA 
Another important factor for the PLS regression model is the number of binary solutions needed for the matrix calibration, the initial number was set considering all the possible combinations and levels where ASP and SA can be found mixed, this is necessary in order to confer discriminating power to the algorithm. The initial number of calibration binary solutions was set at 39 and was optimized up to 13 solutions. The results from both sets of solutions were compared, showing non statistical significance difference after ANOVA test, in the recovery of three levels of ASP+SA standard mixes prepared by sextuple. Finally, calibration matrix used for the PLS model involved only 13 standard solutions, measured at 13 wavelengths. The statistical parameters of the PLS multivariate method showed 4 as the best number of latent variables, this was calculated based on the linearity of the model by the prediction sum of squares (PRESS). Importantly, the goodnessof-fit statistics of the final PLS model showed a good prediction capability of $\mathrm{R}^{2}=0.9984$ and a standard error of the regression of $\mathrm{S}=0.1284$ for ASP. Additionally, in order to prove reproducibility, and not hydrolysis of ASP at room temperature and ambient humidity conditions, the calibration mix was evaluated for five consecutive days. Results showed that there were no statistical significant differences after ANOVA test on the quality parameters of the models.

Once spectrophotometric PLS conditions and sample treatment were established, the method was validated following ICH guidelines ${ }^{[25,26]}$. Selectivity was checked by comparing the analysis of a standards mixes, spiking samples and placebo. It was observed that placebo showed some absorbance at the range of 200-236 nm. However, this aspect did not interfere with the method since this wavelength range was not considered in the model.

As first test, two calibration matrixes were performed using either standards or spiked samples. The results showed negligible difference $(0.02 \%)$ in the slope value between both calibration curves. Moreover, recovery calculation based on interpolation of the systems from one curve using the complementary calibration matrix showed no statistical differences on recovery after ANOVA test (mean recovery $=99.41 \%$ and $98.96 \%$ of ASP using standard and spiked sample curves, respectively).

Results from the proper parameters of the validation are shown on Table 1. Regarding linearity, both standards and samples showed a good linear regression with determination coefficients over 0.99 and no bias. The \%RSD of slopes for ASP and SA ranged less than $0.75 \%$ for both, representing the good fit of individual points to the regression line. Method precision had RSD values of 0.43 and $1.16 \%$ for ASP using standards and samples, respectively. Precision at first day for spiked ASP at $100 \%$, proved RSD values of 0.94 and $0.38 \%$

TABLE 1: SUMMARY OF VALIDATION PARAMETERS FOR ASP*

\begin{tabular}{|c|c|c|c|c|c|}
\hline \multicolumn{6}{|c|}{ Linearity and range from standards $(n=13)$} \\
\hline Standards & $r^{2}$ & $\mathrm{~A} \pm \mathrm{SD}$ & $\mathrm{B} \pm \mathrm{SD}$ & Range $\mu \mathrm{g} / \mathrm{ml}$ & \%RDS ${ }^{\S}$ \\
\hline ASP & 0.9997 & $0.9997( \pm 0.0032)$ & $0.014( \pm 0.1632)$ & $30-70$ & 0.38 \\
\hline SA & 0.9999 & $0.9999( \pm 0.0012)$ & $0.0012( \pm 0.0285)$ & $0-40$ & 0.75 \\
\hline \multicolumn{6}{|l|}{ Samples } \\
\hline ASP & 0.9963 & $0.9926( \pm 0.0166)$ & $0.1585( \pm 0.6732)$ & $36.5-56.5$ & 0.50 \\
\hline \multicolumn{6}{|c|}{ Precision and Recovery $(n=6)$} \\
\hline Standards & Mean recovery (\%) & Recovery SD & Precision, RSD(\%) & & \\
\hline ASP & 99.41 & 0.42 & 0.43 & & \\
\hline SA & 99.75 & 0.61 & 0.61 & & \\
\hline \multicolumn{6}{|l|}{ Samples } \\
\hline ASP & 98.96 & & 1.16 & & \\
\hline \multicolumn{6}{|c|}{ Inter-day and Inter-analyst precision of ASP mean recovery (\%) } \\
\hline & $\begin{array}{l}\text { Repeatability } \\
\text { Day } 1(n=3)\end{array}$ & $\begin{array}{c}\text { Intermediate } \\
\text { precision } \\
\text { Day } 2(n=3)\end{array}$ & $\begin{array}{c}\text { Inter-day repeatability } \\
\text { Day } 1+2(n=6)\end{array}$ & & \\
\hline Analyst 1 & 100.82 & 99.26 & 100.04 & & \\
\hline Analyst 2 & 99.21 & 101.92 & 100.56 & & \\
\hline $\begin{array}{c}\text { Inter-analyst } \\
\text { repeatability }(n=6)\end{array}$ & 100.01 & 100.59 & & & \\
\hline
\end{tabular}


by two analysts while 1.05 and $0.85 \%$ were obtained from two days of analysis. Therefore, precision met the expected values to be validated for inter-analyst and inter-day tests. Moreover, recovery was found in $100.01 \%$ and $100.59 \%$ of ASP for standards and samples, respectively, accomplishing validation criterion.

Sensitivity proves how precise is the method to discriminate small differences in the analyte concentrations. Moreover, in the case of degradation products for which the amount is frequently very small and the acceptable limits are reduced; sensitivity becomes paramount interest to decide whether the pharmaceutical product is still in good condition to be sold. Results for sensitivity system showed that it can discriminate concentrations of 4 and $1 \mu \mathrm{g} / \mathrm{ml}$ for ASP and SA, respectively, whereas in samples the sensitivity was 1.5 and $1.8 \mu \mathrm{g} / \mathrm{ml}$ for ASP and SA. These results demonstrated high sensitivity of the analytical method to quantify accurately close concentrations.

Tolerance was measured $0.2 \mathrm{~nm}$ above established wavelengths in six spiked samples containing $100 \%$ of ASP, where no statistical difference was observed after two way ANOVA test $(\mathrm{P}=0.11)$. Moreover for robustness, absorption spectra were obtained using methanol as dissolving reagent. The results showed similar absorption patterns to anhydrous alcohol. No statistical difference was recorded for recovery of spiked samples dissolved in methanol compared to anhydrous alcohol ( $\mathrm{P}=1.0$ for both analytes).

The last point in the validation method was the stability study of the already prepared standards and tablet samples. Therefore, prepared standard binary solutions were measured fresh and after different time points, always maintained at room temperature (Table 2). The results showed that the ASP recovery did not change until $6 \mathrm{~h}$ after the standard mix was prepared, obtaining a mean recovery of $96.61 \%$ and a RSD of $0.98 \%$. This is important as $6 \mathrm{~h}$ is the lapse time where ASP did not suffer any degradation and can be measured accurately using the model from the PLS regression. Furthermore, stability of developed and Aspirin ${ }^{\circledR}$ commercial tablets was tested through six replicates from each. Results in Table 2 proved SA started to appear after the first hour in Aspirin ${ }^{\circledR}$ samples whereas for developed tablets ASP still stable until $2 \mathrm{~h}$ after sample was prepared. Thus, after $6 \mathrm{~h}$ of storage, an average of $3.7 \%$ of SA is formed in Aspirin ${ }^{\circledR}$ samples.

The results of this method showed great advantages over those techniques that use previous separation of the compounds. Herein, this method is low cost, easy to carry out and to get established as a routine analytical method. Once the calibration matrix is set, uses short time of analysis and low organic volume compared to HPLC technique, and the analytical response of

TABLE 2: APPLICATION OF THE STABILITY INDICATING METHOD FOR ASPIRIN AND SALICYLIC ACID IN STANDARD SOLUTIONS, SYNTHETIC AND ASPIRIN ${ }^{\circledR}$ COMMERCIAL SAMPLES

\begin{tabular}{|c|c|c|c|c|}
\hline aStandard ASP+SA $(n=18)$ & \multicolumn{2}{|c|}{ ASP } & \multicolumn{2}{|c|}{ SA } \\
\hline $\mathbf{h}$ & $\bar{X}$ recovery (\%) & RSD (\%) & $\bar{X}$ recovery (\%) & RSD (\%) \\
\hline 0 & 99.3 & 0.43 & 101.2 & 0.92 \\
\hline 3 & 97.8 & 0.89 & 111.4 & 2.04 \\
\hline 6 & 96.6 & 0.98 & 115.2 & 1.24 \\
\hline 22 & 92.6 & 0.73 & 136.7 & 0.51 \\
\hline ASP & \multicolumn{2}{|c|}{${ }^{\mathrm{b}} \mathrm{ASP}$ developed $(\mathrm{n}=6)$} & \multicolumn{2}{|c|}{${ }^{{ } A S P P I R I N}{ }^{\circledast}(n=6)$} \\
\hline $\mathrm{h}$ & $\bar{X}$ recovery (\%) & RSD (\%) & $\bar{X}$ recovery (\%) & RSD (\%) \\
\hline 0 & 99.3 & 0.40 & 98.7 & 0.77 \\
\hline 1 & 98.3 & 0.69 & 96.2 & 0.82 \\
\hline 2 & 96.4 & 0.88 & 95.6 & 0.21 \\
\hline 4 & 92.9 & 1.42 & 94.1 & 0.78 \\
\hline 6 & 91.9 & 1.25 & 91.1 & 0.66 \\
\hline SA formation & \multicolumn{2}{|c|}{${ }^{\mathrm{b}} \mathrm{ASP}$ developed $(n=6)$} & \multicolumn{2}{|c|}{${ }^{\text {cAspirin }}{ }^{\circledast}(n=6)$} \\
\hline $\mathrm{h}$ & $\bar{X}$ recovery (\%) & RSD (\%) & $\bar{X}$ recovery (\%) & RSD (\%) \\
\hline 0 & 0 & 0 & 0 & 0 \\
\hline 1 & 0 & 0.42 & 0.39 & 0.24 \\
\hline 2 & 0.83 & 0.43 & 1.01 & 0.29 \\
\hline 4 & 2.63 & 0.48 & 2.11 & 0.24 \\
\hline 6 & 4.54 & 0.72 & 3.72 & 0.37 \\
\hline
\end{tabular}


ASP and SA perfectly suits with the purpose of the determination.

This paper demonstrates in depth the high potential of PLS regression in the development of a stabilityindicating method resolving overlapped absorbance spectra in binary unbalanced mixes containing high concentration of ASP and very low quantity of SA. Noteworthy, this method is able to quantify accurately both compounds without prior separation avoiding the use of extra equipment. It is fast and easy to perform, and adaptable as a routine analysis at low cost. This methodology represents a reliable alternative from existing methods to analyse ASP in tablets.

\section{Acknowledgements:}

The authors thank Supporting Grant PAPIIT IN218115 of DGAPA-Universidad Nacional Autónoma de México. Conacyt by the doctoral scholarship granted to Mariana Dolores Hernández

\section{Financial support and sponsorship:}

Nil.

\section{REFERENCES}

1. Miner J, Hoffhines A. The discovery of aspirin's antithrombotic effects. Tex Heart Inst J 2007;34:179-86.

2. Sweetman SC. Martindale: The Complete Drug Reference. London: Pharmaceutical Press; 2007.

3. Carstensen JT, Christopher R. Drug stability, principles and practices. New York: Marcel Dekker Inc; 1990.

4. Miners JO. Drug interactions involving aspirin (acetylsalicylic acid) and salicylic acid. Clin Pharmacokinet 1989;17:327-44.

5. Krivosíková Z, Spustová V, Dzúrik R. A highly sensitive HPLC method for the simultaneous determination of acetylsalicylic, salicylic and salicyluric acids in biologic fluids: pharmacokinetic, metabolic and monitoring implications. Methods Find Exp Clin Pharmacol 1996;18:527-32.

6. Mullangi R, Sharma K, Srinivas NR. Review of HPLC methods and HPLC methods with mass spectrometric detection for direct determination of aspirin with its metabolite(s) in various biological matrices. Biomed Chromatogr 2012;26:906-41.

7. Patel SM, Patel CN, Patel VB. Stability indicating HPLC Method for Simultaneous Determination of Aspirin and Prasugrel. Indian J Pharm Sci 2013;75:413-9.

8. Sena MM, Poppi RJ. N-way PLS applied to simultaneous spectrophotometric determination of acetylsalicylic acid, paracetamol and caffeine. J Pharm Biomed Anal 2004;34:27-34.

9. Martos NR, Díaz AM, Navalón A, De Orbe Payá I, Capitán Vallvey LF. Simultaneous spectrofluorimetric determination of acetylsalicylic acid, codeine and pyridoxine in pharmaceutical preparations using partial least-squares multivariate calibration. J Pharm Biomed Anal 2000;23:837-44.

10. Navalón A, Blanc R, Del Olmo M, Vilchez JL. Simultaneous determination of naproxen, salicylic acid and acetylsalicylic acid by spectrofluorimetry using partial least-squares (PLS) multivariate calibration. Talanta 1999;48:469-75.

11. Schmidt PC, Glombitza Tiibingen BW. Quantitative multicomponent analysis of aspirin and salicylic acid in tablets without separation of excipients by means of principal component regression and a classical least squares algorithm. Trends Anal Chem 1995;14:45-9.

12. Chhonker YS, Pandey CP, Chandasana H, Laxman TS, Prasad YD, Narain VS, et al. Simultaneous quantitation of acetylsalicylic acid and clopidogrel along with their metabolites in human plasma using liquid chromatography tandem mass spectrometry. Biomed Chromatogr 2016;30:466-73.

13. Darwish K, Salama I, Mostafa S, El-Sadek M. Validated stability-indicating reversed-phase-HPLC method for simultaneous determination of orphenadrine citrate, caffeine and aspirin. Chem Pharm Bull 2012;60:1426-36.

14. Porwal PK, Akhalaque Ahmad RA, Chhajed SS, Chatpalliwar VA. Liquid chromatographic method for simultaneous quantitation of clopidogrel, aspirin and atorvastatin in rat plasma and its application to the pharmacokinetic study. J Chromatogr Sci 2015;53:1155-62.

15. Murtaza G, Khan SA, Shabbir A, Mahmood A, Asad MHHB, Farzana K, et al. Development of a UV-spectrophotometric method for the simultaneous determination of aspirin and paracetamol in tablets. Sci Res Essays 2011;6:417-21.

16. Sankar ASK, Vetrichelvan T, Venkappaya D. Simultaneous estimation of ramipril, acetylsalicylic acid and atorvastatin calcium by chemometrics assisted UV spectrophotometric method in capsules. Acta Pharm 2011;61:283-96.

17. Dinç E, Ozdemir A, Baleanu D. Comparative study of the continuous wavelet transform, derivative and partial least squares methods applied to the overlapping spectra for the simultaneous quantitative resolution of ascorbic acid and acetylsalicylic acid in effervescent tablets. J Pharm Biomed Anal 2005;37:569-75.

18. Carstensen JT. Stability of solids and solid dosage forms. J Pharm Sci 1974;63:1-14.

19. ICH QA, Stability Testing of New Drug Substances and Products. Proceedings of the International Conference on Harmonisation. Geneva; 1993.

20. Cartwright AC, Matthew BR. International Pharmaceutical Product Registration. Ellis Horwood Limited; 1994.

21. Verstraeten A, Roets E, Hoogmartens J. Quantitative determination by high-performance liquid chromatography of acetylsalicylic acid and related substances in tablets. J Chromatogr 1987;388:201-16.

22. Kumar SS, Jamadar LD, Bhat K, Musmade PB, Vasantharaju SG, Udupa N. Analytical method development and validation for aspirin. Int J Chem Tech Res 2010;2:389-99.

23. Rubak P, Hardlei TF, Würtz M, Kristensen SD, Hvas AM. Low dose acetylsalicylic acid therapy monitored with ultrahigh performance liquid chromatography. Clin Biochem 2013;46:988-92.

24. Bautista RD, Aberásturi FJ, Jiménez AI, Jiménez F. Simultaneous spectrophotometric determination of drugs in pharmaceutical preparations using multiple linear regression and partial least-squares regression, calibration and prediction methods. Talanta 1996;43:2107-15. 
www.ijpsonline.com

25. Haaland MD, Thomas EV. Partial least squares methods for spectra analysis. Application to simulated and glass spectral data. Anal Chem 1988;60:1202-20.

26. http://www.ema.europa.eu/docs/en_GB/document_library/ Scientific_guideline/2009/09/WC500002662.pdf.
27. https://www.ich.org/fileadmin/Public_Web_Site/ICH Products/Guidelines/Quality/Q2_R1/Step4/Q2_R1 Guideline.pdf. 